\title{
FIMATH -
}

\section{Finanzmathematische Berechnungen für Juristen}

Programm-Autor: Helmut Hoffmann

Mit dieser finanzmathematischen Programmsammlung können nach den Verfahren der einfachen Zinsrechnung und der Zinseszinsrechnung Anfangs-und Endkapital sowie Zinssatz und Laufzeit ermittelt werden.

Den Sonderfall von Jahres- Bruchteilen berechnet das Programm nach der gemischten Zinsrechnung.

Ein Programm zur Berechnung von Auf- und Abzinsungsfaktoren für beliebige Zinssätze und ein Taschenrechner für Nebenrechnungen runden die Hoffmannsche Sammlung ab.

\section{Disketteninhalt}

Auf der für Abonnenten dieser Ausgabe von jur-pc beigelegten Diskette befinden sich folgende Dateien:

FIMATH.EXE

MATHHELP.DBF

MATHHELP.DBT

MATHINST . DBF

READ.ME und README.1ST

Die Datei READ.ME enthält ausführliche Hinweise zu Bedienung und finanzmathematischen wie juristischen Grundlagen der Programme. READ.ME kann mit dem Betriebssystem-Befehl „TYPE“ (type read.me(ENTER)) am Bildschirm gelesen werden - der Bildlauf läßt sich mit 〈CTRL〉 $S$ unterbrechen undit 〈CTRL〉 $Q$ fortsetzen - oder bspw. mit COPY (copy read.me prn〈ENTER〉) auf dem Drucker ausgeben.

\section{Programmstart und Bedienung}

FIMATH kann direkt von der Diskette oder - nach dem Kopieren der Dateien von der Diskette in ein zuvor mit MD angelegtes Verzeichnis der Festplatte (z.B. MD C:/ FIMATH) mit COPY (z.B. copy a:** c:/fimath) - auch von der Festplatte durch die Eingabe

- FIMATH〈ENTER

gestartet werden.

Die Berechnungsergebnisse können auf einem Drucker protokolliert oder zur Weiterverarbeitung in eine ASCII-Datei ausgegeben werden.

\section{Finanzmathematische Grundlagen}

Die Programme der Sammlung gehen - entsprechend der banküblichen Praxis - von jährlicher nachschüssiger Verzinsung aus.

\section{Einfache Zinsen}

Das geltende Recht der Verzugszinsen geht von einfachen Zinsen aus, d.h. die anfallenden Zinsen werden dem Kapital nicht zugeschlagen, sondern getrennt aufaddiert, so daß kein Zinseszinseffekt entsteht. Dies entspricht dem Verbot der Zinseszinsen in $§ 284$ BGB.

\section{Zinseszinsen}

Bei der Zinseszins-Berechnung werden die angelaufenen Zinsen am Ende des Jahres dem Kapital zugeschlagen und in der nächsten Zinsperiode mitverzinst (Zinseszinseffekt).

\section{Gemischte Zinsrechnung}

Läßt sich die Gesamtlaufzeit nicht als ganzzahliges Vielfaches eines Jahres ausdrücken und soll die Zinseszinsmethode Anwendung finden, stellt sich die Frage, ob sich die Zinsansprüche für den Jahres-Bruchteil nach der einfachen Zinsrechnung oder ebenfalls nach der Zinseszins-Methode bestimmen.

Die gemischte Zinsrechnung geht davon aus, daß die Verzinsung für die vollen Jahre nach der Zinseszins-Methode erfolgt, für den Jahres-Bruchteil jedoch nach der einfachen 
Zinsrechnung. Dies entspricht dem üblichen Vorgehen der Banken und liegt auch dem Programm zugrunde.

\section{Konventionen und Formeln}

1. Bankmäßige Zeitberechnung

Bankwesen und Finanzmathematik arbeiten mit folgenden Konventionen:

1 Monat $=30$ Tage

$1 \mathrm{Jahr}=360$ Tage

2. Variablen - Bezeichnung und Bedeutung

$i$ = Zinssatz (nomineller Jahreszins)

$\mathrm{K}_{\mathrm{O}}=$ Anfangskapital (in einer beliebigen Währungseinheit)

$\mathrm{K}_{\mathrm{n}}=$ Endkapital

$\mathrm{n}$ = Laufzeit (in Jahren)

3. Formeln bei jährlicher Vierzinsung

a) Berechnung des Endkapitals

Einfache Zinsrechnung

$\mathrm{K}_{\mathrm{n}}=\mathrm{K}_{0} *(1+\mathrm{n} * \mathrm{i})$

Zinseszinsrechnung $\mathrm{K}_{\mathrm{n}}=\mathrm{K}_{0} *(1+i)^{\mathrm{n}}$

Gemischte Verzinsung

Zwischenrechnung $n_{1}=\operatorname{iNT}(n)$

$\mathrm{n}_{2}=\mathrm{n}-\mathrm{n}_{1}$

Formel

$\mathrm{K}_{\mathrm{n}}=\mathrm{K}_{0}+(1+\mathrm{i})^{\mathrm{n}_{1}} *\left(1+\mathrm{n}_{2} * \mathrm{i}\right)$

b) Berechnung des Anfangskapitals

Einfache Zinsrechnung

$\mathrm{K}_{0}=\mathrm{K}_{\mathrm{n}} /(1+\mathrm{n} * \mathrm{i})$

Zinseszinsrechnung

$\mathrm{K}_{\mathrm{n}}=\mathrm{K}_{0} *(1+\mathrm{i})^{-\mathrm{n}}$

Gemischte Verzinsung

$\mathrm{K}_{0}=\mathrm{K}_{\mathrm{n}} *\left[(1+\mathrm{i})^{\mathrm{n}_{1}} *\left(1+\mathrm{n}_{2} * \mathrm{i}\right)\right]$

c) Berechnung des Zinssatzes

Einfache Zinsrechnung

$\dot{i}=(1 / n) *\left(K_{n} / K_{0}-1\right)$

Zinseszinsrechnung

$\dot{i}=\left(\mathrm{K}_{\mathrm{n}} / \mathrm{K}_{0}\right)^{(1 / \mathrm{n})_{-1}}$

Gemischte Verzinsung

Bei der gemischten Verzinsung ist die Berechnung des Zinssatzes nur durch eine sog. Iteration möglich. Die Gleichung für die Errechnung des Endkapitals läßt sich nicht nach $\mathrm{i}$ auflösen, so $\mathrm{daß}$ es eine Gleichung für den Zinssatz nicht formuliert werden kann.

Das Programm arbeitet nach dem Newton-Verfahren zur Nullstellenbestimmung einer Funktion. Zur Steigerung der Berechnungsgeschwindigkeit wird als Startwert der Iteration nicht ein beliebiger, sondern der mit der Formel der Zinseszinsrechnung ermittelte Wert für den Jahreszins eingesetzt.

$\mathrm{f}(\mathrm{i})=-\mathrm{Kn}+\mathrm{KO} *(1+\mathrm{i})^{\mathrm{n} 1} *(1+\mathrm{n} 2 * \mathrm{i})$

d) Aufzinsungs- und Abzinsungsfaktoren

Dieses Programm errechnet für beliebige Zinssätze die Auf- und Abzinsungsfaktoren und stellt die errechneten Faktoren tabellarisch dar.

auf $z=(1+i)^{n}$

$a b z=(1+i)^{-n}$ 